\title{
POSTURES SOCIODISCURSIVES ET CONQUÊTE DU POUVOIR
}

\author{
Pascal Marchand \& Pierre Ratinaud ${ }^{1}$
}

Les périodes précédant une élection présidentielle sont propices au dévoilement des ambitions personnelles qui conduisent à des stratégies complexes pour apparaître comme une personnalité forte et distincte tout en représentant au mieux le parti dont on brigue l'investiture. Être à la fois soi et les autres implique d'adopter une posture crédible et attractive. L'analyse lexicométrique d'un ouvrage politique collectif intitulé Les 12 travaux de l'opposition : Nos projets pour redresser la France, publié par l'UMP (2014) permet de définir trois postures sociodiscursives, que nous appelons généraliste (être visionnaire), spécialiste (être compétent dans un domaine) et polémiste (être combatif à l'égard des adversaires). On analyse également les risques qu'il y a à trop investir une posture ou à se situer à leur intersection.

La Constitution de la $V^{e}$ République Française, telle qu'elle était voulue par Charles de Gaulle, cherchait à rompre avec un " régime

1 Pascal Marchand est Professeur en Sciences de l'Information et de la Communication à l'Université de Toulouse-Paul Sabatier et rattaché au Laboratoire d'Études et recherches appliquées en Sciences Sociales (Lerass). Pierre Ratinaud est Maître de conférences en Sciences de l'Éducation à l'Université de Toulouse-Jean Jaurès et rattaché au Lerass.

Recherches en communication, $\mathrm{n}^{\circ} 41$ (2014). 
des partis » pour placer le Président au cœur de l'exécutif. Mais cette rencontre particulière d'un homme et de la nation peut-elle se passer des organisations partisanes ? Au lendemain de la seconde guerre mondiale, certainement. Par la suite, c'est assurément moins évident et, aujourd'hui, rien n'est moins sûr. L'histoire de la V $V^{e}$ République est une histoire de groupes autant que de personnages politiques et l'accession aux plus hautes fonctions de l'Etat semble nécessairement passer par la conquête d'un parti ou, du moins, la quête de sa reconnaissance.

Les procédures de désignation $\mathrm{du} /$ de la candidat-e est propre à chaque parti et relève souvent de manœuvres internes qui font la délectation des analystes et commentateurs. La campagne de 2007 a vu le Parti socialiste inaugurer une formule inspirée des élections américaines : les Primaires dites « citoyennes ». Rééditées en 2012, ces Primaires ont vu se confronter des leaders du parti (ou de partis alliés, comme le Parti Radical de Gauche) sur un plateau de télévision et à propos de questions calquées sur l'agenda médiatique.

A droite, on a d'abord été tenté par le mépris pour cette procédure. Puis, on a réalisé qu'elle répondait sans doute à une demande ou, tout du moins, à un « esprit du temps » souvent assimilé à une demande. Quoi qu'il en soit, l'idée s'est progressivement imposée dans les instances de l'UMP $^{1}$ que la procédure des Primaires devait être reprise, quitte à l'adapter. Sans que l'on puisse aujourd'hui savoir si, finalement, ces Primaires seront effectivement mises en place, car des mouvements de résistance existent toujours, on en repère néanmoins des signes avantcoureurs.

L'exercice du débat des primaires n'est pas facile puisque, pour avoir une chance de rassembler le maximum de suffrages dans la consultation qui s'ensuivra, chacun-e doit s'efforcer à la fois d'être le plus apte au consensus et d'apparaître comme la personnalité la plus forte et la plus digne d'incarner la fonction présidentielle. Dans un jeu d'équilibriste entre identité et personnalité, il s'agit donc de « convaincre de ses similitudes tout en démontrant ses différences » (Mange \& Marchand, 2011, p. 87) pour que le débat s'installe sans mettre en péril la cohésion du groupe. C'est, du moins, l'enjeu qui pèse sur les débatteurs. Même si des rapprochements seraient évidemment imaginables, il ne s'agit pas ici de postures énonciatives, ainsi que les définit Alain Rabatel (2007), ni de l'éthos - présentation de soi - décrites par Ruth Amossy (2010), mais

1 Depuis 2002, l'Union pour un mouvement populaire (UMP) est le principal parti politique français de droite. Il est devenu «Les Républicains », parfois abrégé en LR, en mai 2015, à l'initiative de Nicolas Sarkozy. 
de stratégies qui englobent, outre la situation d'interaction, les positions sociales parfois complexes des locuteurs, les représentations sociales des objets discursifs abordés, les images des groupes en présence et de leurs relations (coopération et compétition intra- et intergroupes)... C'est l'ensemble de ces contraintes qui définit les termes du contrat de communication (Ghiglione, 1986, 1989 ; Charaudeau, 1997) - voire ici du contrat de lectorat (Burguet, 2003 ; Veron, 1985) - dont on pose ici l'hypothèse qu'il est susceptible d'amener chacun-e à se définir une posture sociodiscursive qui, le temps du débat, présidera à l'élaboration d'une stratégie rhétorique et au choix d'un registre lexical.

Sur la base d'un corpus d'exemple, nous montrerons comment l'analyse lexicométrique préalable d'un ouvrage politique définit des classes thématiques spécifiques qui peuvent, dans un deuxième temps, être lues en fonction de postures sociodiscursives généralisables à d'autres situations discursives.

\section{Douze travaux, un corpus}

La parution, en septembre 2014, d'un ouvrage coécrit par douze hautes personnalités de l'UMP et intitulé : Les 12 travaux de l'opposition : Nos projets pour redresser la France (Apparu) n'était pas présentée comme un débat pour des Primaires. Cela étant, cet ouvrage comptait parmi ses contributeurs, des personnalités ouvertement déclarées candidates à la Présidence de la République, ou au moins à celle de 1'UMP, généralement vue comme une étape, ou d'autres ayant publiquement indiqué leur soutien à un-e candidat-e. Il ne semble donc pas inopportun de considérer, à titre d'hypothèse, que l'écriture de chacun des douze chapitres (précédés par un prologue, mais sans épilogue) a dû suivre un objectif assez similaire à celui qui est généralement assigné à des Primaires. On est alors autorisé à penser que ce livre doit être traversé de positions, voire même d'oppositions, qui doivent s'exprimer par des lexiques identifiables. Si c'est effectivement le cas, les douze chapitres devraient donner à voir des similitudes et des différences visibles par une analyse des données textuelles, telle que la permet le logiciel Iramuteq $^{l}$, qui reprend notamment la méthode de classification lexicale proposée par Reinert (1983, 1984).

1 Pierre Ratinaud, université de Toulouse : http://www.iramuteq.org 


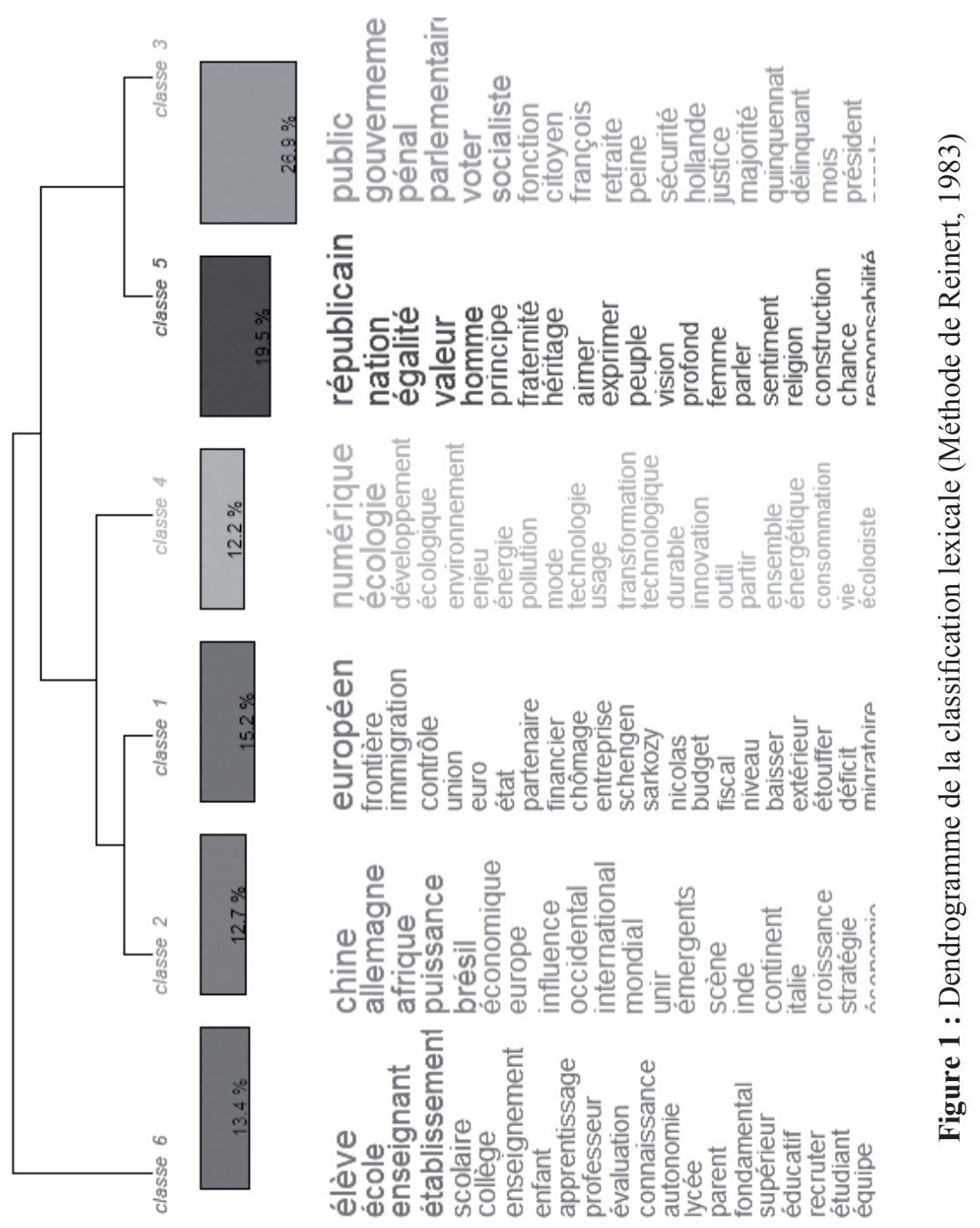




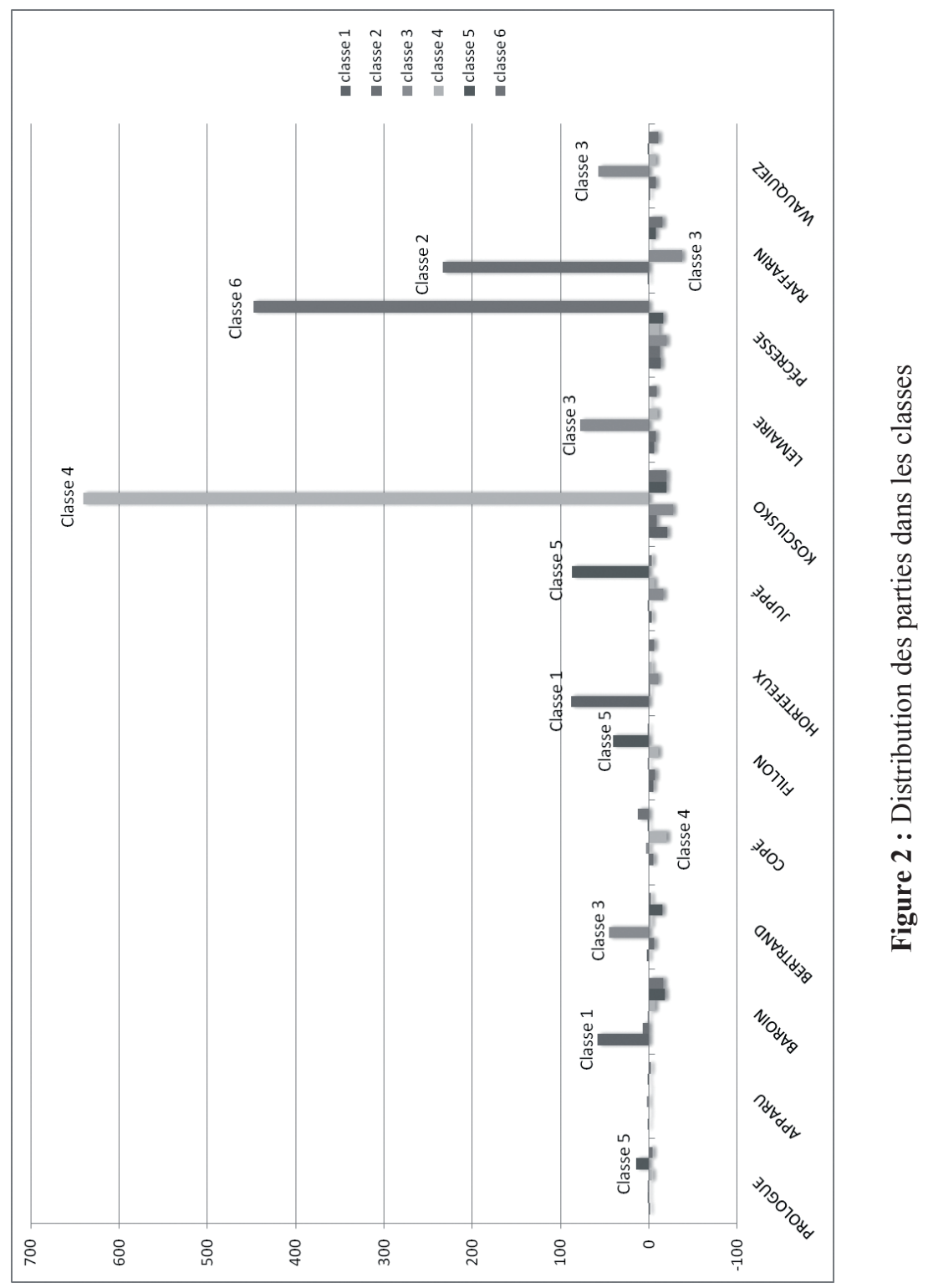


Outre le chapitre introductif, les contributeurs-trices ont été codé-e-s et on pourra donc comparer les lexiques de Benoist Apparu, François Baroin, Xavier Bertrand, Jean-François Copé, François Fillon, Brice Hortefeux, Alain Juppé, Nathalie Kosciusko-Morizet, Bruno Le Maire, Valérie Pécresse, Jean-Pierre Raffarin et Laurent Wauquiez ${ }^{1}$.

L'ouvrage, dont le texte a été fidèlement saisi par nos soins, comprend 45663 mots, ce qui en fait un corpus de petite taille, correspondant à un vocabulaire de 6746 mots différents (53\% étant des hapax) pouvant constituer les colonnes d'un tableau lexical. Ce corpus a ensuite été découpé en 1265 segments de textes d'une longueur moyenne de 36 mots (en respectant la ponctuation) pour constituer les lignes du tableau lexical. Le codage de la présence (1) ou de l'absence (0) d'un mot en colonne dans un segment de texte en ligne constitue la base de la classification du lexique : il s'agit de regrouper les segments de texte dans des classes les plus homogènes possibles au regard des «profils » présentés par les mots du corpus.

Cette analyse dite de " classification hiérarchique descendante » permet ici de classer près de $90 \%$ du corpus en six classes ainsi que le montrent les Figures 1 et 2. La Figure 1 représente le dendrogramme de la classification, sur lequel on a fait figurer les mots les plus significatifs de chaque classe. La Figure 2 représente la dépendance que l'on peut établir entre les classes lexicales et les contributeurs-trices.

\section{Douze travaux, six thématiques}

Six grandes thématiques semblent donc structurer les Douze travaux, que nous pouvons décrire dans l'ordre de leur définition statistique. Les mots les plus corrélés ainsi que les segments de textes les plus significatifs des classes nous permettent les interprétations qui suivent.

\subsection{L'éducation}

\section{(classe $6 \approx 13 \%$ des propos classés)}

C'est la première thématique à se dégager par sa grande cohérence interne. Sans trop de surprise, il s'agit d'une thématique chère à Valérie Pécresse, ancienne Ministre de l'Enseignement supérieur et de la

1 Les chapitres de l'ouvrage se succèdent également dans l'ordre alphabétique des auteurs, ce qui nous renseigne sur le fait qu'un regroupement thématique n'est pas apparu souhaitable aux initiateurs du projet. 
Recherche, puisque $80 \%$ de ses propos se retrouvent dans cette classe. Ce thème de l'Education est, on le sait, l'un de ceux qui favorisent le plus le clivage gauche / droite. Et on notera éventuellement que les segments de textes les plus caractéristiques de cette thématique ne sont pas des propos de Valérie Pécresse mais des phrases de Jean-François Copé :

1. Copé (score $=736.18)$ : Les établissements accueillant les élèves les moins favorisés doivent d'ailleurs bénéficier de dotations leur permettant de recruter les meilleurs enseignants. Je propose un examen des savoirs fondamentaux, en début de CM2, avant l'entrée au collège.

2. Copé $($ score $=693.14)$ : Des enseignants mieux formés, mieux rémunérés et disposant de meilleurs outils, notamment grâce au numérique. Je propose de réaffirmer l'autorité des maîtres et, selon le choix des établissements, d'imposer aux élèves un code vestimentaire qui ferait grandir le sentiment d'appartenance à une communauté scolaire.

3. Pécresse (score $=633.62)$ : Les premiers acteurs de la réforme sont les enseignants. Ce sont eux qui feront bouger les lignes, dans les établissements, au plus près de leurs élèves et de leurs familles. Il faut remettre nos professeurs au centre du système éducatif.

4. Fillon (score $=619.61)$ : Pour garantir l'apprentissage des fondamentaux par tous les élèves, je propose de concentrer l'enseignement élémentaire sur le socle de connaissances (lecture, écriture, calcul...), de vérifier l'acquisition de ces fondamentaux...

5. ... de commencer la scolarité obligatoire dès 5 ans, d'augmenter le temps de présence des enseignants dans leurs établissements. Au collège, ces fondamentaux devront être approfondis - le brevet devenant un certificat d'acquisition de ce socle de connaissances.

6. Pécresse (score $=511.36):$ La réforme des universités m'a aussi permis de travailler avec les enseignants-chercheurs 
sur les qualités mais aussi sur les faiblesses de nos étudiants, lorsqu'à 18-20 ans ils frappent à la porte de nos établissements d'enseignement supérieur.

\subsection{Technologie et le développement durable (classe $4 \approx 12 \%$ des propos classés)}

$84 \%$ des propos de Nathalie Kosciusko-Morizet se retrouvent dans cette classe, ce qui ne surprend pas outre mesure de la part d'une ancienne Secrétaire d'État chargée de l'Écologie (2009), puis de la Prospective et du développement de l'Économie numérique (20092010), devenue enfin Ministre de l'Écologie, du Développement durable, des Transports et du Logement (2010 -2012). L'auteure reste ici dans son domaine de compétence.

1. Kosciusko $($ score $=617.32)$ : Au premier rang de ces transformations figurent aujourd'hui l'écologie et le numérique. Ce sont les deux grands mouvements de fond qui changent nos sociétés et nos modes de vie; ce sont peut-être les deux grands enjeux universels du moment.

2. Kosciusko (score $=597.82):$ L'économie de l'ingéniosité. Ce point de frottement de l'écologie et du numérique, ce sont toutes les technologies du développement durable et tout ce que l'on appelle également les nouvelles technologies «vertes » (les « Green IT »)...

3. ... qui produisent des outils numériques plus économes en énergie et moins polluants. On a là tout un ensemble d'entreprises au statut hybride, qui sont à la fois soucieuses d'environnement et de très haut niveau technologique.

4. Kosciusko (score $=583.00)$ : Tout cela s'applique tout particulièrement au numérique et à l'écologie. Révolutionnaires, oui, parce que la numérisation d'une partie considérable des activités humaines est déjà engagée et va s'accélérer; parce que le développement durable va devenir une exigence de vie commune à tous. 
5. Kosciusko (score $=579.01)$ : Les nouvelles technologies ont deux vocations : dynamiser l'ensemble des activités économiques, et accompagner la croissance verte. Le numérique va servir de levier à l'ensemble des activités économiques, et, là où il rencontre l'écologie, il doit contribuer à l'émergence d'une nouvelle économie.

\subsection{Europe et immigration (classe 1 15\% des propos classés)}

Ces deux thématiques apparaissent liées ici, notamment par une problématique de frontières et de risque (contrôle, maîtrise...), et concernent essentiellement Brice Hortefeux mais également François Baroin. Le premier a été un Ministre remarqué, d'abord del'Immigration, de l'Intégration, de l'Identité nationale et du Codéveloppement (20072009), puis de l'Intérieur, de l'Outre-mer, des Collectivités territoriales et de l'Immigration (2009-2011). On ne s'étonnera donc pas que 88\% de ses propos se retrouvent ici (flux, migratoire, immigré, clandestin...). Quant à François Baroin, ses fonctions de Ministre de l'Intérieur et de l'Aménagement du territoire (2007), puis de Ministre du Budget, des Comptes publics, de la Fonction publique et de la Réforme de l'État (2007-2011) et enfin de l'Économie, des Finances et de 1'Industrie (2011-2012) lui confèrent également une pertinence certaine dans le registre de cette classe qu'il investit pour 58\% de ses propos, moins sur la question de l'immigration que sur celle de l'économie européenne (emploi, compétitivité, déficit...).

1. Hortefeux (score $=403.33$ ) : Or, depuis l'adoption du Pacte européen sur l'immigration et l'asile sous la présidence de Nicolas Sarkozy en 2008 qui avait donné une véritable impulsion à la politique migratoire en Europe, l'Union européenne n'est pas parvenue à atteindre les objectifs qu'elle s'était fixés.

2. Hortefeux (score $=328.98)$ : Dans le domaine de la politique d'immigration, l'Union européenne n'a pas suffisamment agi. Pourtant, c'est à ce niveau précis que nous avons le pouvoir de mieux contrôler les flux migratoires et il existe des leviers d'action qui n'ont pas été pleinement exploités. 
3. Hortefeux (score $=317.80)$ : Concrètement, comme l'a proposé Michel Barnier, l'Union européenne doit créer un corps européen de garde-frontière capable d'organiser et de piloter les opérations de renfort aux frontières extérieures. Nous devons parallèlement établir un dialogue régulier avec les pays sources et de transit.

4. Bertrand ( $($ core $=306.07):$ Si l'adoption d'un système pénal commun aux vingt-huit états membres de l'union européenne semble illusoire à court terme compte tenu des différentes traditions et de la règle de l'unanimité qui s'applique à ces matières, nous devons promouvoir une coordination renforcée entre les services nationaux.

5. Hortefeux $($ score $=298.52)$ : Avec 182595 premiers titres de séjour délivrés en 2011, la France a aussi eu un niveau d'immigration légale inférieur à celui de ses grands partenaires européens...

6. Baroin (score $=293.55)$ : La solidarité européenne s'est encore renforcée le 2 août 2012 avec la décision symbolique de la banque centrale européenne d'intervenir «sans limite » dans le rachat des titres de dette des états membres de l'union européenne en difficulté.

7. Baroin (score $=292.52)$ : Les responsables politiques européens, conduits par Nicolas Sarkozy et Angela Merkel, ont dû élaborer en urgence des solutions audacieuses, structurées autour de deux objectifs principaux, à savoir la garantie financière des états et du système financier d'une part, afin notamment de protéger l'épargne des citoyens européens, et l'assainissement des finances publiques de l'autre.

\subsection{Relations internationales (classe $2 \approx 13 \%$ des propos classés)}

Cette thématique est essentiellement investie par Jean-Pierre Raffarin, puisque l'ancien Premier ministre y développe près de 57\% de ses propos, mais également par François Baroin, pour les mêmes raisons que dans la classe précédente. 
1. Raffarin (score $=445.56)$ : Cette fragilité européenne, cette confusion sur les finalités du projet européen surviennent alors que l'émergence de "pays-continents », par exemple et pas seulement les BRICS (Brésil, Russie, Inde, Chine, Afrique du Sud), modifie profondément l'ordre international.

2. Raffarin (score $=375.71)$ : En 2012, la production industrielle des émergents a dépassé celle des pays développés. En 2025, $61 \%$ de la population mondiale se trouvera en Asie et le Pib total de la Chine et de l'Inde dépassera celui des sept plus grandes économies mondiales (le G7) d'ici 2025.

3. Raffarin (score $=364.11)$ : Pis encore, concernant l'Inde, nous arrivons loin derrière l'Allemagne, le Royaume-Uni, l'Italie et la Belgique avec $1 \%$ de part de marché ! Notre perte d'influence sur la scène internationale est une évidence (...).

4. Baroin (score $=340.74)$ : Alors qu'elle pourrait bénéficier de relais de croissance en vendant aux pays les plus dynamiques, la France perd du terrain dans certains grands pays émergents, comme la Chine, où les parts de marché de l'Allemagne sont aujourd'hui plus de quatre fois plus élevées que les nôtres.

5. Raffarin (score $=340.64)$ : L'Europe n'est plus un démultiplicateur de puissance pour la France et le couple franco-allemand est grippé en raison du décrochage économique de notre pays. La France n'est plus en pointe sur les grands dossiers internationaux...

\subsection{Les valeurs républicaines (classe $5 \approx 19,5 \%$ des propos classés)}

Les deux anciens Premiers ministres, Alain Juppé et François Fillon, se retrouvent dans cette classe, dont le vocabulaire investit également le prologue de l'ouvrage.

1. Fillon (score $=261.06)$ : Pour l'égalité des chances, il y a en France un idéal républicain cher à notre nation et dont la réalité s'affadit : la possibilité qu'un Français, quelle que soit 
sa situation sociale d'origine, puisse, par ses seuls talents, ses seuls mérites, s'élever aux plus hautes places.

2. Juppé $($ score $=248.86):$ L'histoire, la culture, la langue... notre bien commun, ce sont aussi des valeurs et des principes politiques. S'intégrer à la nation française, c'est faire siens ces valeurs et ces principes.

3. Fillon $($ score $=231.34)$ : Pour cela, nous devons nous mobiliser pour revivifier en urgence ses principes : libertéégalité-fraternité. Liberté " chérie » par les révolutionnaires, la liberté est le premier mot des Républicains, inspiratrice de la Révolution de 1789 ; sans elle, il n'y a pas de République.

4. Juppé ( $($ core $=220.67):$ Je m'étonne souvent - étonnement mêlé de beaucoup d'admiration - de constater l'actualité de notre devise républicaine : liberté, égalité, fraternité... En trois mots, les pères fondateurs de la France contemporaine ont tout dit.

5. Hortefeux $($ score $=220.16):$ Il y a également lieu de réformer le code de la nationalité. La nationalité française n'est pas un dû, c'est une récompense au terme d'une démarche volontaire d'intégration, la démonstration d'un attachement manifeste pour la France républicaine, les valeurs et les principes qu'elle défend.

6. Fillon ( $(\mathrm{score}=219.00)$ : Il faut affermir l'égalité juridique, mais dénuée d'une idéologie qui refuse les distinctions; il faut encourager partout l'égalité des chances mais bannir l'égalitarisme ; assumer la solidarité, que justifie l'appartenance à une même nation, mais la fonder sur la responsabilité.

7. Juppé (score $=215.84)$ : Intégrer, dans notre modèle républicain, c'est, tout en respectant les différences, offrir à ceux qui nous rejoignent de partager le bien commun qui unit la nation parce-que nous l'avons reçu de ceux qui nous ont précédés. 
8. Juppé $($ score $=215.60)$ : Toujours dans le bien commun de la nation française s'inscrit le principe démocratique, c'est-à-dire l'affirmation que le pouvoir vient du peuple et que la volonté populaire s'exprime par le suffrage, censitaire d'abord, puis universel.

9. Juppé $($ score $=210.14)$ : Dès lors que la religion musulmane et ses fidèles s'intègrent dans le modèle républicain à la française et respectent notre vision commune de la laïcité, ils ont naturellement toute leur place parmi nous.

10. Fillon ( score $=190.55)$ : Vous devez l'aider aussi : c'est le contrat républicain. Pour la neutralité républicaine. Au nom de l'égalité, la république doit se caractériser par la neutralité : c'est la conséquence du principe fondamental de laïcité, auquel, comme les Français, je suis attaché.

\subsection{La politique (classe $3 \approx 27 \%$ des propos classés de l'ouvrage)}

C'est la thématique la plus représentée et s'y retrouvent les aspects les plus polémiques des relations politiques, celles qu'affectionnent les commentateurs et interviewers, mettant en scènes des conflits de bassecour et des courses de chevaux (Patterson, 1980). Ce sont surtout Bruno Le Maire, Laurent Wauquiez et Xavier Bertrand qui investissent ce vocabulaire'.

1. Baroin (score $=178.00)$ : Contrairement aux affirmations répétées de l'actuelle majorité socialiste, le précédent gouvernement de François Fillon n'a pas laissé derrière lui une situation budgétaire dégradée. Il a fait le choix audacieux, nécessaire et assumé d'engager une politique de relance face à la récession mondiale, au prix de déficits accrus.

2. Lemaire $($ score $=161.72)$ : Quelle hypocrisie de la part de tant de parlementaires socialistes, qui pourtant avaient pris l'engagement de ne plus cumuler et ont voté le texte de loi,

1 Si Jean-François Copé investit ce lexique pour un tiers de ses propos, sa liaison avec la classe n'atteint pas le seuil de significativité statistique requis. 
mais se sont présentés en tête de liste aux dernières élections municipales de 2014.

3. Baroin (score $=159.35)$ : La seconde erreur, également due à une vision idéologique de l'économie, a été de détricoter systématiquement les réformes structurelles engagées par le gouvernement de François Fillon et sa majorité parlementaire, au lieu de les approfondir.

4. Bertrand $($ score $=157.43)$ : Pour parvenir à une réforme structurelle de la sécurité intérieure dont notre pays a grandement besoin, il nous faut relever huit défis. Seul un changement complet dans la manière d'appréhender la question permettra de redonner de la légitimité et de l'efficacité à notre système pénal.

5. Prologue (score $=156.06)$ : Or ce sont bien les positionnements électoralistes, sans réelle ambition sinon celle d'être réélu, qui pèsent aujourd'hui dans le rapport des citoyens à la politique. Les décisions prises ces deux dernières années par les gouvernements de François Hollande en sont une éclatante et effarante illustration.

6. Copé $($ score $=154.74)$ : On notera au passage que la principale contribution de François Hollande à ce chantier au cours de son quinquennat aura été la réouverture du tribunal de grande instance de Tulle en 2013... La loi pénale de Christiane Taubira devra être immédiatement abrogée et le nombre de places de prison substantiellement augmenté...

7. Lemaire (score $=147.38)$ : Il y a quelques mois, avec d'autres parlementaires de toutes tendances politiques, $\mathrm{j}$ 'ai participé à une initiative en ce sens. Ma proposition de loi sur la révolution démocratique a été soumise à discussion sur un site internet dédié : www.parlement-et-citoyens.fr.

8. Copé $($ score $=143.94)$ : En théorie, le président de la République peut s'appuyer sur une majorité parlementaire et un gouvernement stables. La $\mathrm{V}^{\mathrm{e}}$ République lui donne tous les leviers institutionnels pour conduire sa politique. 
9. Wauquiez (score $=142.45)$ : Il me paraît également important de garantir l'indépendance des politiques par rapport aux lobbies privés. Quand un parlementaire a d'autres engagements professionnels, il faut les rendre publics, avec notamment la liste des clients et honoraires.

10. Baroin (score $=139.80)$ : Début 2014, le crédit d'impôt « compétitivité emploi » (CICE) est entré en application, reprenant le modèle de la réforme de la "TVA sociale » votée par la précédente majorité conduite par François Fillon et détricotée en 2012, mais en y ajoutant une touche de bureaucratie qui en sape partiellement l'efficacité.

\section{Douze travaux, trois postures}

Si l'analyse des données textuelles définit six classes lexicales, chacune délimitant une thématique susceptible d'être investie par les contributeurs-trices, un regard synthétique sur la classification nous amène finalement à identifier différentes postures sociodiscursives. Cette proposition repose sur une analyse du discours d'inspiration psychosociale.

En contexte électoral, Charaudeau (2013, pp. 7-10) distingue des stratégies de parole destinées à se démarquer des adversaires et reposant sur une " logique symbolique », exaltant les valeurs, et sur une " logique pragmatique », affirmant la crédibilité. Nous sommes tentés de rapprocher ces "logiques » de la théorie psychosociale de l'identification de l'action (Wegner, Vallacher, Macomber, Wood \& Arps, 1984). Cette théorie rend compte du fait que les gens peuvent identifier une même action à des niveaux variables, du plus concret au plus abstrait, du plus particulier au plus général. On observe généralement que l'expertise amène à donner un sens plus général aux actions, à les identifier au plus haut niveau d'abstraction possible. On peut effectivement repérer ici une articulation possible entre logiques discursives et niveaux d'identification de l'action en définissant des postures sociodiscursives que nous appellerons généraliste, spécialiste et polémiste. 


\subsection{Les généralistes}

Privilégiant la « logique symbolique », ils identifient l'action politique à un haut niveau d'abstraction. Ce sont les idéologues, garants de la doxa du groupe. On retrouve essentiellement ici les anciens Premiers ministres, Alain Juppé et François Fillon. Leur légitimité repose sur leur façon d'incarner les valeurs et l'éthique du groupe. On pourrait rapprocher cette posture de ce que Charaudeau (2005, p. 163) décrit comme imaginaire sociodiscursif de la «tradition ». S'il s'agit moins de la quête de l'origine ou d'un ressource aux sources, il s'agit néanmoins de s'ancrer dans une lignée historique et de puiser dans la « matrice idéologique de la droite » (Charaudeau, 2013, p. 176).

Assez logiquement, le prologue de l'ouvrage puise également dans ce vocabulaire symbolique-abstrait, ainsi que le montre son segment le plus représentatif :

Pourtant, nous croyons qu'un renouveau est possible. Nous croyons à la responsabilité politique. Nous croyons au travail, un travail collectif, de long terme, qui préparera le redressement mais saura aussi recréer un élan collectif, ressusciter l'espoir et la foi en demain (Charaudeau, 2013).

\subsection{Les spécialistes}

Ils sont, à l'opposé des précédents, dans une identification de l'action politique à un niveau plus concret: l'univers sensible et le règne de l'évidence. Valérie Pécresse, Nathalie Kosciusko-Morizet, Brice Hortefeux, Jean-Pierre Raffarin et, moins spécifiquement, François Baroin sont dans ce cas. Relevant d'un ethos de crédibilité et mobilisant un imaginaire de "modernité », volontiers économiste ou technologiste (Charaudeau, 2005, p. 166), ils se présentent comme des experts, ont assis leur notoriété sur un domaine de spécialité, et leur légitimité repose sur leurs compétences revendiquées et leur bilan valorisé à la tête d'un ministère.

\subsection{Les polémistes}

Ils échappent un peu à la dichotomie des « logiques » comme aux niveaux d'identification de l'action. Des travaux postérieurs auront à déterminer s'ils se situent dans un intermédiaire. Ils fondent leur 
légitimité sur leur capacité à générer et alimenter le conflit politique, en l'orientant de préférence vers le hors-groupe : créer l'unité en stigmatisant l'adversaire. Il s'agit bien là d'une attitude polémique, par opposition à une attitude didactique (Courdesses, 1971), et au sens que lui donnent Albert et Nicolas : " prise de parole primordialement caractérisée par l'attaque et l'opposition voire la disqualification systématique de la parole d'un autre (adversaire réel ou imaginé) - ce que nous pourrions appeler l'usage vectorisé de la violence verbale » (2010, p. 17).

Il s'agit à la fois de mobiliser des réflexes émotionnels et identitaires. D'un point de vue émotionnel, c'est bien à la violence symbolique et à un "discours dicté par les affects et les pulsions émotionnelles » (Kerbrat-Orecchioni, 1980, p. 20) ${ }^{1}$ que l'on est renvoyé. En termes identitaires, on retrouve la psychologie sociale des relations intergroupes : "À la limite, les membres d'un groupe n'ont rien d'autre en commun que le fait de ne pas partager les caractéristiques du horsgroupe. [...] L'identité sociale repose (alors) carrément sur les épaules du hors-groupe » (Allen, Wilder \& Atkinson, 1983 cité par LorenziCioldi \& Dafflon, 1999, pp. 136-137). Pour reprendre une déclaration fameuse d'Henri Tajfel, "nous sommes ce que nous sommes parce qu'ils ne sont pas ce que nous sommes » $(1979$, p. 183). On retrouve ici principalement Bruno Le Maire, Laurent Wauquiez et Xavier Bertrand.

On notera que la plupart des contributeurs à l'ouvrage tendent à privilégier l'une de ces trois postures, à l'exception de Benoist Apparu ou Jean-François Copé, mais il semble que ce soit pour des raisons différentes.

Benoist Apparu choisit d'investir un domaine de compétence particulier, celui de l'aménagement du territoire et des collectivités locales. Si ce vocabulaire n'apparaît pas assez homogène, structuré et partagé pour générer une classe thématique, on peut le rattacher aux « spécialistes » :

1 Ce qui ne signifie pas, et on rejoindra Plantin (2003), que les deux autres postures ne comportent ni passion, ni violence. Mais c'est sans doute dans la posture polémiste qu'elles s'expriment de la façon la plus directe dès lors qu'il s'agit essentiellement « d'occuper le terrain et de se mettre en scène » (Albert et Nicolas, op. cit.). Il s'agit donc essentiellement d'une question d'habillage rhétorique et on pourrait sans doute retrouver, derrière les postures généralistes, spécialiste et polémiste, des éléments d'ethos, de logos et de pathos. 
1.... des régions plus puissantes moins par la taille que par l'octroi de compétences pleines et entières (voir ci-dessus), leur autonomie fiscale, une fusion des conseils régionaux et départementaux et des intercommunalités qui recevraient les compétences des conseils municipaux et se verraient déléguer la compétence sociale.

2. Depuis plusieurs années, deux thèses s'affrontent. Les départementalistes, qui préconisent les grandes régions et le maintien du conseil départemental, s'opposent aux régionalistes, qui souhaitent supprimer le conseil départemental avec un redécoupage à la marge des régions.

3. Ces régions nouvelles recevraient les compétences actuelles des régions et des départements (avec dotations et fiscalité correspondantes) mais aussi, pour ne pas dire surtout, obtiendraient la capacité de définir les modalités d'exercice de ces compétences sur la base d'objectifs fixés avec l'État.

Quant à Jean-François Copé, le fait qu'il n'apparaisse pas très fortement liés aux classes lexicales vient au contraire de ce qu'il associe les postures, notamment en faisant porter la polémique sur les valeurs, là où les polémistes dénoncent généralement une action gouvernementale. C'est alors sans doute celui qui puise le plus dans la " matrice idéologique de la droite » (Charaudeau, op. cit.), notamment avec un recours particulièrement fort à la valeur de liberté, associée à une tonalité polémiste :

1. J'aime la France quand elle incarne la liberté ! À mes yeux, reprendre ce combat pour la liberté de l'homme est une urgence : c'est renouer avec les fils de notre histoire et ouvrir de nouveaux chemins pour la jeunesse de France.

2. Combien de fois faudra-t-il rappeler à la gauche qu'un pays sans sécurité, c'est un pays sans liberté ? Une autorité à réinventer. Pour garantir cette liberté, pour en finir avec la peur qui grandit dans nos cités et nos villages, il faut reconstruire une nouvelle autorité. 
3. Pour eux, on n'en fait jamais assez pour l'égalité et toujours trop pour la liberté. Pour eux, l'ultra-égalitarisme est un paradis et l'ultralibéralisme un enfer. C'est absurde ! Les deux doivent être combattus.

Disqualifier l'adversaire sur des valeurs consensuelles contribue à sortir le conflit politique d'une opposition doctrinaire, idéologique ou même technique, pour le placer sur le terrain de la morale, ce qui constitue une caractéristique du débat politique des années 2000 (Marchand, 2010). Mais, outre la liberté, ce sont également les notions de travail et de famille qui jouent comme référence identitaire de la droite :

Demain, il faudra couper la bride et reprendre comme leitmotiv la célèbre formule du président Pompidou : « Arrêtons d'emmerder les Français. » Libérons le travail. Finissons-en une bonne fois pour toutes avec les 35 heures en laissant les négociations sur le temps de travail se faire dans les entreprises.

La réaffirmation du caractère universel de la politique familiale (aider toutes les familles, à raison du nombre d'enfants et non des revenus) sera primordiale.

\section{Remarques conclusives}

La typologie que nous proposons ici, en trois postures sociodiscursives, généraliste, spécialiste et polémiste, permet d'interpréter une analyse lexicométrique, tout en confirmant des analyses antérieures issues de méthodologies bien différentes et en convoquant des univers disciplinaires distincts.

Précisons immédiatement qu'il n'est pas question de renvoyer à des traits de caractère permanents de telle ou telle personne, ni d'affirmer qu'elle EST généraliste, spécialiste ou polémiste ${ }^{1}$, mais de constater que, dans ce contexte et pour cette demande précise, les unes et les autres ont adopté une posture discursive qui nous indique leur

1 On a néanmoins pu observer qu'en politique, le discours des sujets peu engagés traduit souvent un faible niveau d'identification de l'action (préoccupation quotidienne, concrète et égocentrée), tandis que les sujets très engagés identifient les actions à un niveau plus haut (sociétal, abstrait et conceptuel). 
façon de se représenter la situation et ses implications. On a pu voir que des stratégies convoquant simultanément ces postures étaient possibles, mais elles présentent le risque de la caricature.

Notre analyse montre qu'il peut y avoir, à un moment pré-électoral, lorsque se désigne celui ou celle qui portera la parole du groupe devant la consultation nationale, une répartition des rôles pour, à défaut d'apparaître unis, donner l'impression qu'on joue la même pièce. Cela ne dit rien, en revanche, des chances que peut avoir telle ou telle personne d'accéder au leadership du parti ou à la présidence du pays et l'avenir nous confirmera sans doute que la conquête du pouvoir prend d'autres voies que le débat d'idées et le développement de contenus, avantageusement remplacés par des sondages et cotes de popularité.

Le peu d'intérêt des commentateurs politiques pour les contenus politiques a effectivement été pointé par Le Bart, Leroux et Ringoot (2014), pour qui la vie politique « ne serait plus principalement régie par les relations institutionnelles entre titulaires de rôles mais par des relations entre personnalités politiques dotées de styles et de tempéraments singuliers. Ces personnalités se meuvent sous le regard à la fois privilégié et distancié des journalistes, dont le principal mérite est alors d'avoir été témoins de ce qui se passe en coulisse ». Face à ce " relatif renoncement des journalistes politiques à directement parler politique », les hommes et femmes politiques peuvent être tenté-e-s d'abandonner les contenus pour privilégier des artifices de pure communication ou des facilités identitaires.

Pour l'analyste du discours politique, il faut alors chercher les situations pertinentes à analyser et elles ne sont finalement pas si fréquentes. Avec ses Douze travaux, l'UMP en a certainement fourni une. Certes, cet ouvrage n'a bénéficié que d'un traitement médiatique superficiel, tout juste limité à en commenter l'arrivée, à rendre compte de courts instants de la conférence de presse qui l'a marquée, et de rappeler les relations de coopération et de compétition entre les contributeurs-trices. Et puis on en est revenu aux véritables événements de la rentrée politique : les succès en librairie de Valérie Trierweiller et d'Eric Zemmour. 


\section{Références}

Albert, L., \& Nicolas, L. (2010). Introduction. Le " pacte » polémique : enjeux rhétoriques du discours de combat. Dans L. Albert \& L. Nicolas (Éd.), Polémique et rhétorique, coll. Champs linguistiques (pp. 17-48). Bruxelles : De Boeck.

Allen, V. L., Wilder, D. A., \& Atkinson, M. L. (1983). Multiple group membership and social identity. Dans T. R. Sarbin \& K. E. Scheibe (Éd.), Studies in Social Identity (pp. 92-115). New York : Praeger.

Amossy, R. (2010). La présentation de soi. Ethos et identité verbale. Paris : Presses Universitaires de France.

Apparu, B. (2014). Les 12 travaux de l'opposition : Nos projets pour redresser la France. Paris : Flammarion.

Burguet, A. (2003). Rappel immédiat d'un article de presse: Effets du contrat de lectorat et de l'accord avec le texte. Nouvelle Revue de Psychologie Sociale, 3, 228-236.

Charaudeau, P. (1997). Le discours d'information médiatique : La construction du miroir social. Paris : Nathan.

Charaudeau, P. (2005). Le discours politique. Les masques du pouvoir. Paris : Vuibert.

Charaudeau, P. (2013). La conquête du pouvoir. Opinion, Persuasion, Valeurs, les discours d'une nouvelle donne politique. Paris : L'Harmattan.

Courdesses, L. (1971). Blum et Thorez en mai 1936 : Analyses d'énoncés. Langue Française, 9, 22-33.

Ghiglione, R. (1986). L'homme communiquant. Paris : Armand Colin.

Ghiglione, R. (1989). Je vous ai compris ou l'analyse des discours politiques. Paris : Armand Colin.

Kerbrat-Orecchioni, C. (1980). La polémique et ses définitions. Dans C. KerbratOrecchioni \& N. Gelas (Éd.), Le discours polémique, Centre de recherches linguistiques et sémiologiques (pp. 3-40). Lyon : PUL.

Le Bart, C., Leroux, P., \& Ringoot, R. (2014). Les livres de journalistes politiques. Sociologie d'un passage à l'acte. Mots, Les langages du politique, 104, 5-18.

Lorenzi-Cioldi, F., \& Dafflon, A.-C. (1999). Rapports entre groupes et identité sociale. Dans J.-L. Beauvois, N. Dubois \& W. Doise (Éd.), La construction sociale de la personne (pp. 131-145). Grenoble : PUG.

Mange, J., \& Marchand, P. (2011). Convaincre de ses similitudes tout en démontrant ses différences : un exemple d'analyse automatique des débats internes au PS. Dans L. Baugnet \& T. Guilbert (Éd.), Discours en contextes, coll. Curapp (pp. 87-101). Paris : PUF.

Marchand, P. (2010). De l'affrontement partisan à la violence symbolique : la déclaration de politique générale dans la $\mathrm{V}^{\mathrm{e}}$ République. Parlement [s], 14, 49-66.

Marchand, P., \& Ratinaud, P. (2012). L'analyse de similitude appliquée aux corpus textuels : les primaires socialistes pour l'élection présidentielle française (septembreoctobre 2011). Actes des 11eme Journées internationales d'Analyse statistique des Données Textuelles (JADT) (pp. 687-699).

Micheli, R. (2010). Qu'est-ce qu'une polémique affective ? Réflexion sur les liens entre la polémique et la construction discursive de l'émotion. Dans L. Albert \& L. Nicolas (Éd.), Polémique et rhétorique, coll. Champs linguistiques (pp. 351-362). Bruxelles : De Boeck.

Patterson, T. (1980). The Mass Media Election. New York : Praeger Publishers. 
Plantin, C. (2003). Des polémistes aux polémiqueurs. Dans G. Declercq, M. Murat \& J. Dangel (Éd.), La parole polémique, coll. Colloques, congrès, conférences sur l'époque moderne et contemporaine (pp. 377-408). Paris : Honoré Champion.

Rabatel, A. (2007). Les enjeux des postures énonciatives et de leur utilisation en didactique. Education \& Didactique, 1(2), 89-116.

Reinert, M. (1983). Une méthode de classification descendante hiérarchique : application à l'analyse lexicale par contexte. Les Cahiers de l'analyse des données, 8(2), 187-198.

Tajfel, H. (1979). Individuals and groups in social psychology. British Journal of Social Psychology, 18, 183-190.

Veron, E. (1985). L'analyse du contrat de lecture. Dans Les médias : expériences et recherches actuelles. Paris : IREP.

Wegner, D.M., Vallacher, R.R., Macomber, G., Wood, R., \& Arps, K. (1984). The Emergence of Action. Journal of Personality and Social Psychology, 46(2), 269279. 